\title{
Endoscopic ultrasound-guided pancreatic duct drainage using a fine-gauge balloon catheter
}

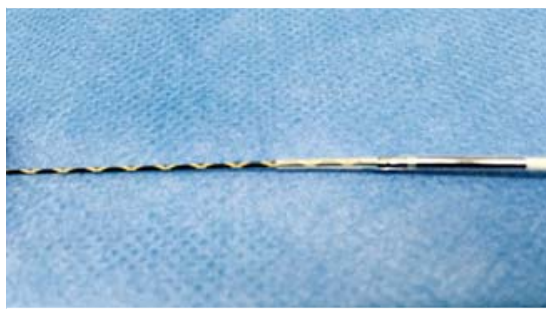

Fig. 1 The novel fine-gauge balloon catheter (REN biliary dilation catheter; KANEKA, Osaka, Japan). The top of this balloon catheter is only $3 \mathrm{Fr}$ and tapered, and is coaxial with the guidewire.

Pancreatic duct drainage under endoscopic retrograde cholangiopancreatography $(E R C P)$ is an established method to treat chronic pancreatitis. However, a pancreatic duct stone is sometimes impacted in the main pancreatic duct. Therefore, pancreatic duct drainage may sometimes be challenging. Recently, various techniques under endoscopic ultrasound guidance have been reported, including biliary drainage or pancreatic duct drainage (EUS-PD) [1-4]. During an EUS-PD procedure, tract dilation is one of the challenging steps. To dilate the tract, electrocautery dilation can be easily performed compared with graded dilation because the parenchyma in chronic pancreatitis is sometimes hard.

Recently, a fine-gauge balloon catheter has been available in Japan (REN biliary dilation catheter; KANEKA, Osaka, Japan) ( $\triangleright$ Fig. 1). The top of this balloon catheter is only $3 \mathrm{Fr}$ and is tapered. In addition, this balloon is coaxial with the guidewire [5]. We herein describe technical tips for EUS-PD using this balloon catheter ( $\downarrow$ Video 1 ).

The echoendoscope was inserted into the stomach, and the main pancreatic duct was identified. The main pancreatic duct was punctured using a 19-G needle (Sono Tip Pro Control 19G; MediGlobe $\mathrm{GmbH}$, Rosenheim, Germany), and the contrast medium was injected ( Fig.2a). Then, a 0.025-inch guide-

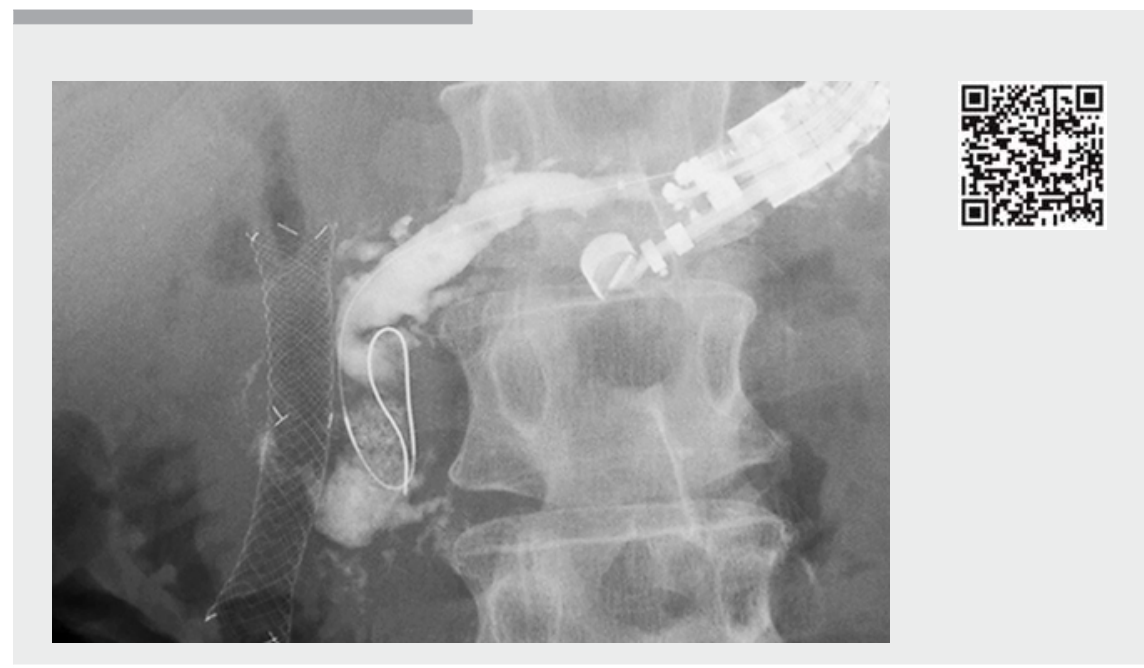

Video 1 The main pancreatic duct is punctured using a 19-G needle, and the contrast medium is injected. The 0.025 -inch guidewire is inserted into the main pancreatic duct. Then, the main pancreatic duct and stomach wall are dilated using fine-gauge balloon catheter. Finally, a plastic stent is deployed from the main pancreatic duct to the stomach.
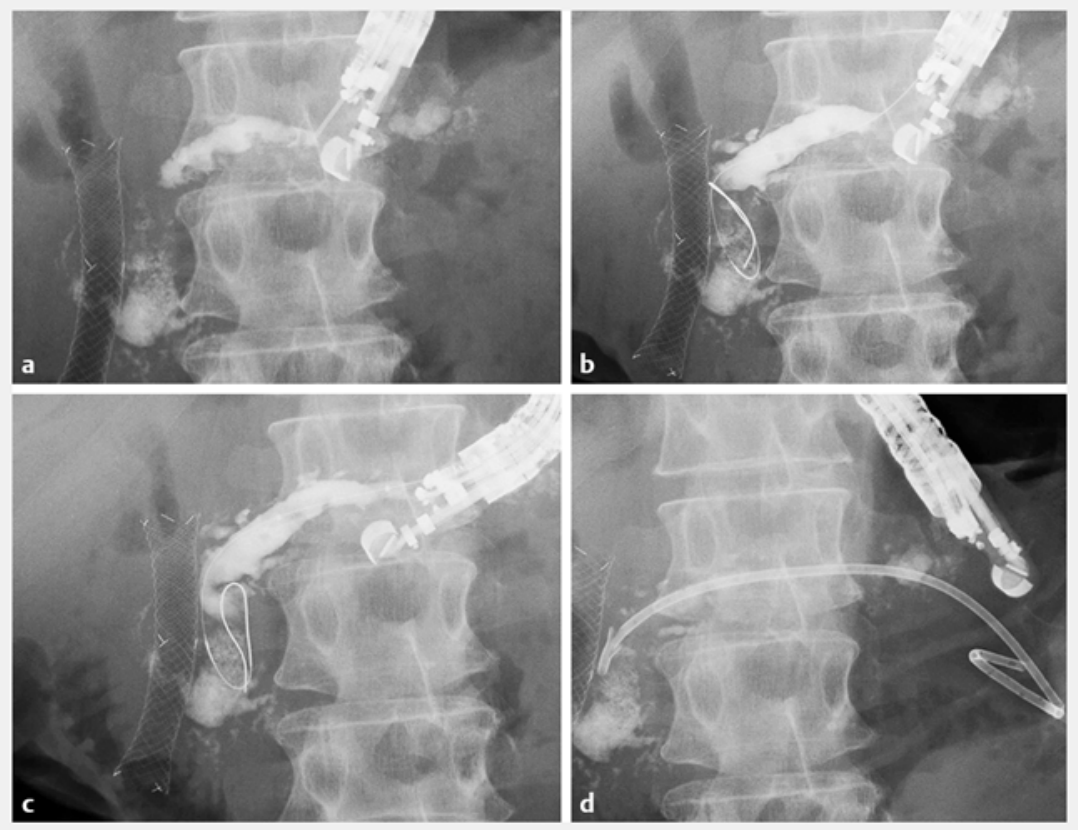

- Fig. 2 Fluoroscopy images. a The contrast medium was injected through a 19-G needle. b A 0.025 -inch guidewire was inserted into the main pancreatic duct. c The main pancreatic duct and stomach wall were dilated using the fine-gauge balloon catheter. $\mathbf{d}$ A plastic stent was deployed from the main pancreatic duct to the stomach. 
wire was inserted into the main pancreatic duct through the needle ( $\triangleright$ Fig. 2 b). Next, the fine-gauge balloon catheter was inserted and successfully dilated the stomach and pancreatic duct walls ( Fig. 2c). Finally, a plastic stent (Gadelius Medical Co, Ltd, Tokyo, Japan) was successfully deployed without any adverse events ( $\triangleright$ Fig. 2 d).

This fine-gauge balloon catheter may have clinical impact not only for use during ERCP but also as a dilation device in various drainage techniques under EUS guidance.

Endoscopy_UCTN_Code_TTT_1AS_2AD

Competing interests

None

The authors

Takeshi Ogura, Nobu Nishioka, Masanori Yamada, Kazuya Ueshima, Kazuhide Higuchi 2nd Department of Internal Medicine, Osaka Medical College, Osaka, Japan
Corresponding author

\section{Takeshi Ogura, MD}

2nd Department of Internal Medicine, Osaka Medical College, 2-7 Daigakuchou,

Takatsukishi, Osaka 569-8686, Japan

Fax: +81-72-6846532

oguratakeshi0411@yahoo.co.jp

\section{References}

[1] Adler JM, Sethi A. Interventional endoscopic ultrasonography in the pancreas. Gastrointest Endosc Clin N Am 2018; 28: 569- 578

[2] Park JK, Woo YS, Noh DH et al. Efficacy of EUS-guided and ERCP-guided biliary drainage for malignant biliary obstruction: prospective randomized controlled study. Gastrointest Endosc 2018; 88: 277-282

[3] Siddiqui UD, Levy ML. EUS-guided transluminal interventions. Gastroenterology 2018; 154: $1911-1924$

[4] Law R, Baron TH. Endoscopic ultrasoundguided gallbladder drainage. Gastrointest Endosc Clin N Am 2018; 28: 187- 195

[5] Amano M, Ogura T, Onda S et al. Prospective clinical study of endoscopic ultrasoundguided biliary drainage using novel balloon catheter (with video). J Gastroenterol Hepatol 2017 ; 32 : $716-720$

\section{Bibliography}

DOI https://doi.org/10.1055/a-0861-9738

Published online: 25.3.2019

Endoscopy 2019; 51: E145-E146

(c) Georg Thieme Verlag KG

Stuttgart · New York

ISSN 0013-726X

\section{ENDOSCOPY E-VIDEOS}

https://eref.thieme.de/e-videos

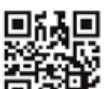

Endoscopy E-Videos is a free access online section, reporting on interesting cases and new

techniques in gastroenterological endoscopy. All papers include a high quality video and all contributions are freely accessible online.

This section has its own submission website at https://mc.manuscriptcentral.com/e-videos 